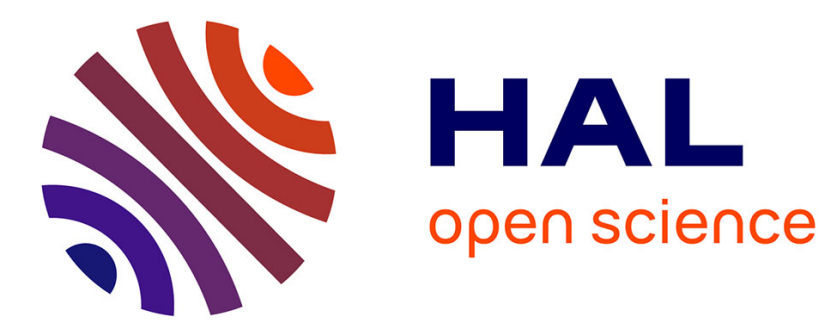

\title{
An arbitrary-order discontinuous skeletal method for solving electrostatics on general polyhedral meshes
}

Daniele Antonio Di Pietro, Bernard A Kapidani, Ruben A Specogna, Francesco A Trevisan

\section{- To cite this version:}

Daniele Antonio Di Pietro, Bernard A Kapidani, Ruben A Specogna, Francesco A Trevisan. An arbitrary-order discontinuous skeletal method for solving electrostatics on general polyhedral meshes. IEEE Transactions on Magnetics, 2017, 53 (6), pp.1-4. 10.1109/TMAG.2017.2666546 . hal-01399505

\section{HAL Id: hal-01399505 \\ https://hal.science/hal-01399505}

Submitted on 19 Nov 2016

HAL is a multi-disciplinary open access archive for the deposit and dissemination of scientific research documents, whether they are published or not. The documents may come from teaching and research institutions in France or abroad, or from public or private research centers.
L'archive ouverte pluridisciplinaire HAL, est destinée au dépôt et à la diffusion de documents scientifiques de niveau recherche, publiés ou non, émanant des établissements d'enseignement et de recherche français ou étrangers, des laboratoires publics ou privés. 


\title{
An arbitrary-order discontinuous skeletal method for solving electrostatics on general polyhedral meshes
}

\author{
Daniele A. Di Pietro ${ }^{1}$, Bernard Kapidani ${ }^{2}$, Ruben Specogna ${ }^{2}$, Francesco Trevisan ${ }^{2}$ \\ ${ }^{1}$ University of Montpellier, Institut Montpelliérain Alexander Grothendieck, 34095 Montpellier, France \\ ${ }^{2}$ Polytechnic Department of Engineering and Architecture (DPIA), Università di Udine, 33100 Udine, Italy
}

\begin{abstract}
We present a numerical method named Mixed High Order (MHO) to obtain high order of convergence for electrostatic problems solved on general polyhedral meshes. The method, based on high-order local reconstructions of differential operators from face and cell degrees of freedom, exhibits a moderate computational cost thanks to hybridization and static condensation that eliminate cell unknowns. After surveying the method, we first assess its effectiveness for three-dimensional problems by comparing for the first time its performances with classical conforming finite elements. Moreover, we emphasize the algebraic equivalence of MHO in the lowest-order with the analog formulation obtained with the Discrete Geometric Approach or the Finite Integration Technique.
\end{abstract}

Index Terms-Poisson problem, electrostatics, high order, polyhedral meshes

\section{INTRODUCTION}

$\mathbf{I}$ $\mathrm{N}$ the past few years, the interest in discretization methods for diffusive problems on general polyhedral meshes has considerably grown, see for example [1] and references therein. Polyhedral mesh generators are currently being developed and once they are going to be available, they will provide more flexibility in element shapes. This flexibility, in turn, should yield to easier techniques for adaptive mesh refinement, derefinement and non-overlapping domain decomposition with non-matching grids. In particular, the non-conforming-like refinement - as the subgridding proposed in [2] - and the adaptive coarsening strategy [3] are particularly appealing.

We consider the problem of seeking the electric displacement vector field $d: \Omega \rightarrow \mathbb{R}^{3}$ and the scalar potential field $v: \Omega \rightarrow \mathbb{R}$ in a polyhedral domain $\Omega \subset \mathbb{R}^{3}$ such that

$$
\begin{aligned}
\varepsilon^{-1} d+\nabla v & =0 & & \text { in } \Omega, \\
\nabla \cdot d & =\rho_{s} & & \text { in } \Omega, \\
v & =0 & & \text { on } \partial \Omega,
\end{aligned}
$$

where $\rho_{s}$ is the volumetric source charge density and $\varepsilon$ is the electric permittivity. We assume that $\varepsilon$ is constant on each element $T$ of a polyhedral mesh $\mathcal{T}_{h}$ of the domain $\Omega$, and we denote its value $\varepsilon_{T}$.

This paper presents the Mixed High-Order (MHO) method of [4], which is able to obtain high order convergence in the solution of (1) on arbitrary polyhedral meshes. This paper aims at assessing the performance on three dimensional problems by comparing with classical conforming finite elements in terms of accuracy vs. computational time. The paper also emphasizes the analogies, in the lowest order case corresponding to the polynomial degree $k=0$, with the Discrete Geometric Approach (DGA) [2]. In particular, this novel

Manuscript received Oct 12, 2016; accepted August 18, 2017. Date of current version April 22, 2017. Corresponding author: R. Specogna (e-mail: ruben.specogna@uniud.it. The work of D. Di Pietro was funded by Agence Nationale de la Recherche project ANR-15-CE40-0005-01.

Color versions of one or more of the figures in this paper are available online at http://ieeexplore.iee.org.

Digital Object Identifier 10.1109/TMAG.2013.2281076 method is algebraically equivalent (up to the choice of a scalar parameter in the stabilisation term) to the mixed-hybrid geometric formulation described in [5]. The MHO method bears also some similarities with the Discontinuous Galerkin (DG) [6], but it presents a higher convergence rate for a given polynomial degree. Contrary to DG, the degrees of freedom are attached to both the faces and the elements of the mesh. Moreover, cell-based unknowns may be easily eliminated by element-wise static condensation.

The paper is organized as follows. In Section II we survey the MHO method and show how to reinterpret the DGA as the MHO method in the lowest-order. Section III presents the comparison of results provided by the $\mathrm{MHO}$ with respect to the finite elements on a test case with analytical solution. Finally, in Section IV, some conclusions are drawn.

\section{THE MiXed High-ORDER METHOD}

The starting point of the MHO method is the classical weak formulation of problem (1): Find $(d, v) \in D \times V$ such that, for all $(\tau, u) \in D \times V$,

$$
\begin{aligned}
\int_{\Omega} \varepsilon^{-1} d \cdot \tau-\int_{\Omega} v(\boldsymbol{\nabla} \cdot \tau) & =0 \\
\int_{\Omega}(\boldsymbol{\nabla} \cdot d) u & =\int_{\Omega} \rho_{s} u,
\end{aligned}
$$

where $D$ denotes the space of square-integrable vector-valued functions whose divergence is also square integrable, while $V$ contains square-integrable scalar-valued functions. The main idea is to define some degrees of freedom (DOFs) in each element to be able to locally reconstruct the divergence operator and the displacement $d$. These reconstructions are used in (2) to write discrete counterparts of each term of (2).

Let a polynomial degree $k \in \mathbb{N}$ be fixed, and consider a mesh element $T \in \mathcal{T}_{h}$. The local space of DOFs for the displacement is defined as the following polynomials

$$
\underline{D}_{T}^{k}:=\left(\varepsilon_{T} \boldsymbol{\nabla} \mathbb{P}^{k}(T)\right) \times \mathbb{P}^{k}\left(\mathcal{F}_{T}\right),
$$

see Fig. 1, where $\mathbb{P}^{k}\left(\mathcal{F}_{T}\right)$ is the space of polynomials of degree $k$ over the boundary of $T$ that are possibly discontinuous at the 


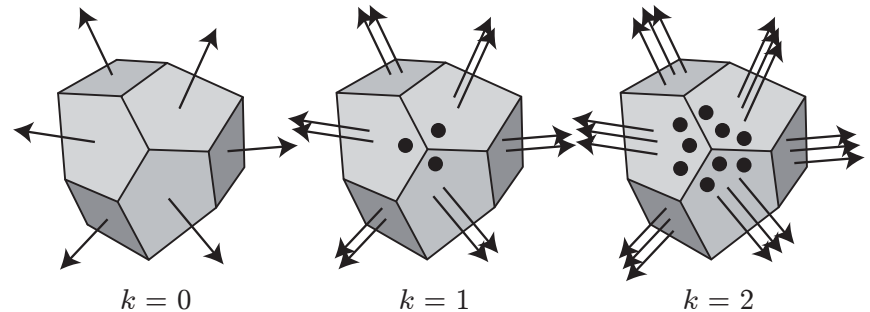

Fig. 1: Local degrees of freedom in $\underline{D}_{T}^{k}$ for $k \in\{0,1,2\}$.

edges shared by the faces of $T$. Element-based and face-based DOFs represent, respectively, polynomial moments of $d$ inside $T$ and polynomial moments of the outward normal component of $d$ on $F$. We remark that element-based DOFs are present only for $k \geqslant 1$. For the collection the total DOFs in $\underline{D}_{T}^{k}$, we use the underlined notation $\underline{\tau}_{T}=\left(\tau_{T}, \tau_{\partial T}\right)$, where $\tau_{T}$ contains the vector-valued element-based DOFs, while $\tau_{\partial T}$ contains the scalar-valued face-based DOFs (which can be interpreted as the polynomial moments of the outward normal component of the displacement).

We define the discrete divergence operator $\mathcal{D}_{T}^{k}: \underline{D}_{T}^{k} \rightarrow$ $\mathbb{P}^{k}(T)$ such that, for all $\underline{\tau}_{T} \in \underline{D}_{T}^{k}$ and all $q \in \mathbb{P}^{k}(T)$,

$$
\int_{T}\left(\mathcal{D}_{T}^{k} \underline{\tau}_{T}\right) q=-\int_{T} \tau_{T} \cdot \nabla q+\int_{\partial T} \tau_{\partial T} q
$$

Equation (3) resembles an integration by parts formula where the role of the displacement inside $T$ and on its boundary $\partial T$ is played by $\tau_{T}$ and $\tau_{\partial T}$, respectively.

By similar principles, the displacement reconstruction operator $\mathcal{R}_{T}^{k}: \underline{D}_{T}^{k} \rightarrow \varepsilon_{T} \nabla \mathbb{P}^{k+1}(T)$ is such that, for all $\underline{\tau}_{T} \in \underline{D}_{T}^{k}$ and all $w \in \mathbb{P}^{k+1}(T)$,

$$
\int_{T} \mathcal{R}_{T}^{k} \underline{\mathcal{\tau}}_{T} \cdot \nabla w=-\int_{T}\left(\mathcal{D}_{T}^{k} \underline{\mathcal{\tau}}_{T}\right) w+\int_{\partial T} \tau_{\partial T} w
$$

Notice that, for all $\underline{\tau}_{T}, \mathcal{R}_{T}^{k} \underline{\tau}_{T}$ is a polynomial one degree higher than the element-based DOFs $\tau_{T}$.

For all $T \in \mathcal{T}_{h}$, the local contribution $\int_{T} \varepsilon^{-1} d \cdot \tau$ to the first term in (2a) is approximated by the bilinear form $\mathrm{m}_{T}$ on $\underline{D}_{T}^{k} \times \underline{D}_{T}^{k}$ such that

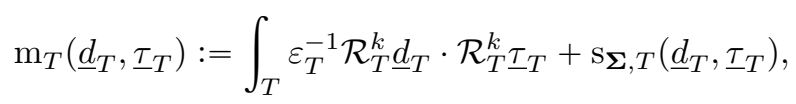

with stabilization bilinear form given by

$$
\begin{aligned}
& \mathrm{s}_{\boldsymbol{\Sigma}, T}\left(\underline{d}_{T}, \underline{\tau}_{T}\right) \\
& \quad:=\int_{\partial T} \gamma_{\partial T}\left(\mathcal{R}_{T}^{k} \underline{d}_{T} \cdot \boldsymbol{n}_{\partial T}-d_{\partial T}\right)\left(\mathcal{R}_{T}^{k} \underline{\tau}_{T} \cdot \boldsymbol{n}_{\partial T}-\tau_{\partial T}\right),
\end{aligned}
$$

where $\boldsymbol{n}_{\partial T}$ is the vector field representing the face normals on $\partial T$ pointing out of $T$ and

$$
\gamma_{\partial T}:=h_{T}\left(\varepsilon_{T} \boldsymbol{n}_{\partial T} \cdot \boldsymbol{n}_{\partial T}\right)^{-1}
$$

with $h_{T}$ diameter of $T$. This stabilization is mandatory for the bilinear form $\mathrm{m}_{T}$ to be positive-definite (SPD). The idea behind (4) is to penalize in a least-square sense the difference between two quantities that both represent the normal component of the displacement on $\partial T$.
We next introduce the global space $\breve{D}_{h}^{k}$ of fully discontinuous DOFs for the displacement as well as its subspace $\underline{D}_{h}^{k}$ with continuous face-based DOFs:

$$
\begin{gathered}
\underline{\check{D}}_{h}^{k}:=\underset{T \in \mathcal{T}_{h}}{\chi} \underline{D}_{T}^{k}, \\
\underline{D}_{h}^{k}:=\left\{\underline{\tau}_{h} \in \underline{\underline{D}}_{h}^{k} \mid \forall F \in \mathcal{F}_{T_{1}} \cap \mathcal{F}_{T_{2}}, \tau_{\partial T_{1} \mid F}+\tau_{\partial T_{2} \mid F}=0\right\} .
\end{gathered}
$$

In practice, this is easily achieved in the usual assembling process by considering the same DOFs for the face shared by two elements.

The approximation of the scalar potential $v$ is sought in

$$
V_{h}^{k}:=\mathbb{P}^{k}\left(\mathcal{T}_{h}\right) .
$$

The MHO method reads: Find $\left(\underline{d}_{h}, v_{h}\right) \in \underline{D}_{h}^{k} \times V_{h}^{k}$ such that for all $\left(\underline{\tau}_{h}, u_{h}\right) \in \underline{D}_{h}^{k} \times V_{h}^{k}$ it holds

$$
\begin{aligned}
\mathrm{m}_{h}\left(\underline{d}_{h}, \underline{\tau}_{h}\right)-\int_{\Omega}\left(\mathcal{D}_{h}^{k} \underline{\tau}_{h}\right) v_{h} & =0, \\
\int_{\Omega}\left(\mathcal{D}_{h}^{k} \underline{d}_{h}\right) u_{h} & =\int_{\Omega} \rho_{s} u_{h},
\end{aligned}
$$

where $\mathrm{m}_{h}$ is obtained by the usual element-by-element assembly and $\mathcal{D}_{h}^{k}$ is set equal to $\mathcal{D}_{T}^{k}$ applied to the restriction of $\underline{\tau}_{h}$ inside each element $T \in \mathcal{T}_{h}$. Convergence as $h^{k+1}$ for the displacement and as $h^{k+2}$ for the potential is proved in [4].

\section{A. Hybridization to obtain a SPD system matrix}

In the numerical results section we use a reformulation of (6) to obtain a SPD sparse system matrix (on the contrary, (6) is a saddle point problem). At the continuous level, the idea is to eliminate the displacement $d$ in (1) and consider the following classical primal formulation where the potential $v$ is the only unknown: Find $v \in U$ such that, for all $u \in U$,

$$
\mathrm{a}(v, u):=\int_{\Omega} \varepsilon \nabla v \cdot \nabla u=\int_{\Omega} \rho_{s} u,
$$

where $U$ contains square-integrable, finite energy functions which comply with the boundary condition (1c).

At the discrete level, the local elimination of the displacement is performed by enforcing the continuity of interface DOFs in $\underline{D}_{h}^{k}$ by Lagrange multipliers (which can be interpreted as in [5] as traces of the potential) and inverting inside each element $T \in \mathcal{T}_{h}$ the local constitutive laws expressed by (6a); cf. [7] for the details.

Let us introduce the following space of hybrid DOFs:

$$
\underline{V}_{h}^{k}:=V_{h}^{k} \times \mathbb{P}^{k}\left(\mathcal{F}_{h}\right) .
$$

For a generic element of $\underline{V}_{h}^{k}$ we use the underlined notation $\underline{v}_{h}=\left(v_{h},\left(v_{F}\right)_{F \in \mathcal{F}_{h}}\right)$. The discrete counterpart of $v$ regarded as an element of $U$ is sought in the following subspace $\underline{V}_{h, 0}^{k}$ of $\underline{V}_{h}^{k}$ incorporating the homogeneous Dirichlet condition on $\partial \Omega$ :

$$
\underline{V}_{h, 0}^{k}:=\left\{\underline{v}_{h} \in \underline{V}_{h}^{k} \mid v_{F}=0 \quad \forall F \in \partial \Omega\right\} .
$$

Let us again concentrate on one element $T \in \mathcal{T}_{h}$. We denote by $\underline{V}_{T}^{k}$ the restriction of $\underline{V}_{h}^{k}$ to $T$ and, for all $\underline{v}_{T} \in \underline{V}_{T}^{k}$, we let $v_{\partial T}$ be the broken polynomial function on $\partial T$ such that $v_{\partial T \mid F}=v_{F}$ for all $F \in \mathcal{F}_{T}$, so that $\underline{v}_{T}=\left(v_{T}, v_{\partial T}\right)$, 

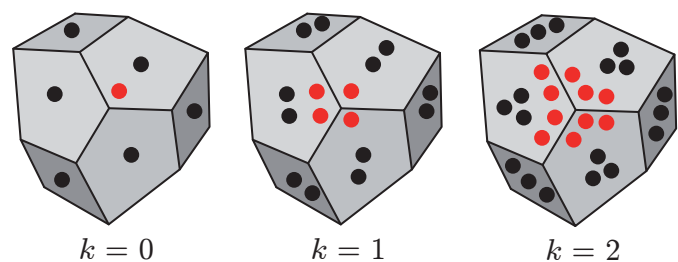

Fig. 2: Local degrees of freedom in $\underline{V}_{T}^{k}$ for $k \in\{0,1,2\}$. Degrees of freedom represented by red dots can be eliminated locally by the static condensation procedure described in [7].

see Fig. 2. From the potential DOFs in $\underline{V}_{T}^{k}$, we can define a reconstruction of displacement DOFs in $\underline{D}_{T}^{k}$ through the potential-to-displacement mapping operator $\underline{\boldsymbol{s}}_{T}^{k}: \underline{V}_{T}^{k} \rightarrow \underline{D}_{T}^{k}$ such that, for all $\underline{v}_{T} \in \underline{V}_{T}^{k}$ and all $\underline{\tau}_{T} \in \underline{D}_{T}^{k}$,

$$
\mathrm{m}_{T}\left(\underline{\boldsymbol{\varsigma}}_{T}^{k} \underline{v}_{T}, \underline{\tau}_{T}\right)=-\int_{T} v_{T}\left(\mathcal{D}_{T}^{k} \underline{\tau}_{T}\right)+\int_{\partial T} v_{\partial T} \tau_{\partial T} .
$$

A high-order reconstruction of the potential can then be defined through the operator $\mathcal{P}_{T}^{k+1}: \underline{V}_{T}^{k} \rightarrow \mathbb{P}^{k+1}(T)$ such that, for all $\underline{v}_{T} \in \underline{V}_{T}^{k}$,

$$
-\varepsilon_{T} \boldsymbol{\nabla} \mathcal{P}_{T}^{k+1} \underline{v}_{T}=\mathcal{R}_{T}^{k}\left(\underline{\varsigma}_{T}^{k} \underline{v}_{T}\right), \int_{T}\left(\mathcal{P}_{T}^{k+1} \underline{v}_{T}-v_{T}\right)=0 .
$$

For a given $\underline{v}_{T} \in \underline{V}_{T}^{k}, \mathcal{P}_{T}^{k+1} \underline{v}_{T}$ is a polynomial one degree higher than the element based DOFs $v_{T} \cdot \mathcal{P}_{T}^{k+1} \underline{v}_{T}$ can be computed directly from the hybrid DOFs in $\underline{v}_{T}$ solving the following problem: For all $w \in \mathbb{P}^{k+1}(T)$,

$$
\begin{aligned}
& \int_{T} \varepsilon_{T} \boldsymbol{\nabla} \mathcal{P}_{T}^{k+1} \underline{v}_{T} \cdot \boldsymbol{\nabla} w \\
& \quad=\int_{T} \varepsilon_{T} \boldsymbol{\nabla} v_{T} \cdot \boldsymbol{\nabla} w+\int_{\partial T}\left(v_{\partial T}-v_{T}\right) \varepsilon_{T} \boldsymbol{\nabla} w \cdot \boldsymbol{n}_{\partial T} .
\end{aligned}
$$

Consider the following approximation of (7): Find $\left(\underline{d}_{h}, \underline{v}_{h}\right) \in \underline{\underline{D}}_{h}^{k} \times \underline{V}_{h, 0}^{k}$ such that, for all $T \in \mathcal{T}_{h}$,

$$
\underline{d}_{T}=-\underline{\boldsymbol{\varsigma}}_{T}^{k} \underline{v}_{T},
$$

and, for all $\underline{u}_{h} \in \underline{V}_{h, 0}^{k}$,

$$
\mathrm{a}_{h}\left(\underline{v}_{h}, \underline{u}_{h}\right):=\sum_{T \in \mathcal{T}_{h}} \mathrm{a}_{T}\left(\underline{v}_{T}, \underline{u}_{T}\right)=\int_{\Omega} \rho_{s} u_{h},
$$

where the local bilinear form $\mathrm{a}_{T}$ on $\underline{V}_{T}^{k} \times \underline{V}_{T}^{k}$ is

$$
\begin{aligned}
\mathrm{a}_{T}\left(\underline{v}_{T}, \underline{u}_{T}\right):=\int_{\Omega} \varepsilon_{T} \boldsymbol{\nabla} \mathcal{P}_{T}^{k+1} \underline{v}_{T} \cdot \boldsymbol{\nabla} \mathcal{P}_{T}^{k+1} \underline{u}_{T} \\
+\mathbf{s}_{\boldsymbol{\Sigma}, T}\left(\underline{\boldsymbol{s}}_{T}^{k} \underline{v}_{T}, \underline{\boldsymbol{\varsigma}}_{T}^{k} \underline{u}_{T}\right) .
\end{aligned}
$$

It can be proved that $\underline{d}_{h} \in \underline{D}_{h}^{k}$ and, with $v_{h} \in V_{h}^{k}$ the broken polynomial function obtained from element-based DOFs in $\underline{v}_{h}$, $\left(\underline{d}_{h}, v_{h}\right) \in \underline{D}_{h}^{k} \times V_{h}^{k}$ solves (6).

\section{B. Link with DGA or FIT}

The DGA of [2] is equivalent to the MHO formulation (6) in the lowest-order case (i.e. $k=0$ ). For the equivalence to work, we have first to assume that, for every mesh element $T \in \mathcal{T}_{h}$, there exists a point $\boldsymbol{x}_{T}$ (i.e. the dual node $\tilde{n}$ ) with respect to which $T$ is star-shaped.
The only difference between the two approaches is in the stabilization parameter $\gamma_{\partial T}$ which, in the case of DGA, is such that for all $F \in \mathcal{F}_{T}$ (compare with (5))

$$
\gamma_{\partial T \mid F}^{\mathrm{dga}}=\left(3 \operatorname{dist}\left(\boldsymbol{x}_{T}, F\right)\right)^{-1} \varepsilon_{T}^{-1}\left(\boldsymbol{x}_{F}-\boldsymbol{x}_{T}\right) \cdot\left(\boldsymbol{x}_{F}-\boldsymbol{x}_{T}\right),
$$

where $\boldsymbol{x}_{F}$ denotes the barycenter of $F$.

\section{NUMERICAL RESULTS}

The implementation of the proposed method is based on the primal form (8). The size of the linear system to solve is further reduced by locally eliminating element-based DOFs by static condensation (represented in red in Figure 2), see [7] for details. Therefore, accounting for the strong enforcement of Dirichlet boundary conditions, a matrix of size $N_{\text {dof }} \times N_{\text {dof }}$ is obtained, where $N_{\text {dof }}=\operatorname{card}\left(\mathcal{F}_{h}^{\mathrm{i}}\right) \times\left(\begin{array}{c}k+2 \\ k\end{array}\right)$ and $\operatorname{card}\left(\mathcal{F}_{h}^{\mathrm{i}}\right)$ is the number of faces in the interior of $\mathcal{T}_{h}$.

The performance of the method is assessed on a problem for which an analytical solution is available. That is, we consider an electrostatic problem in the unit cube $\Omega=(0,1)^{3}$ subject to homogeneous Dirichlet boundary conditions. The charge density $\rho_{s}$ is selected so that the exact solution is $v=\sin (\pi x) \sin (\pi y) \sin (\pi z)$. We evaluate the performances of MHO for polynomial orders $0 \leqslant k \leqslant 4$ by solving the problem on five isotropic tetrahedral meshes obtained as the refinement of the coarsest one. All the sparse linear systems are solved with the algebraic multigrid solver AGMG [8] by stopping the iterations once the relative residual reaches $1 \cdot 10^{-9}$. The computations are performed on a laptop equipped with an Intel Core i7-3720QM processor clocked at $2.60 \mathrm{GHz}$ and $16 \mathrm{~Gb}$ of RAM.

We consider, as a first error measure, the error in the energy norm defined as $e_{\mathrm{en}}:=\left\|\varepsilon^{1 / 2} \nabla_{h}\left(v-\mathcal{P}_{h}^{k+1} \underline{v}_{h}\right)\right\|$, where $v$ denotes the exact solution and $\mathcal{P}_{h}^{k+1}$ is equal to $\mathcal{P}_{T}^{k+1}$ applied to the restriction of $\underline{v}_{h}$ for all $T \in \mathcal{T}_{h}$. We also monitor the approximation of the electrostatic energy $E:=\frac{1}{2} \mathrm{a}(v, v)-\int_{\Omega} \rho_{s} v$ defined as $E_{h}:=\frac{1}{2} \mathrm{a}_{h}\left(\underline{v}_{h}, \underline{v}_{h}\right)-\int_{\Omega} \rho_{s} v_{h}$. Since most global quantities of interest, such as the capacitance, may be obtained from energy, this second error measure is very important in electromagnetic applications.

We remark that we also include the computational time defined as the total wall time needed for the simulation (i.e. not just CPU time), including the pre-processing (mesh generation and creation of mesh incidences), the assembly of the sparse matrix, the solution of the linear system, and the post-processing (electrostatic energy, energy error and, above all, data storage for visualization).

Figures 3 and 4 show the convergence in energy norm $e_{\mathrm{en}}$ with respect to the mesh density $h$ and the number of degrees of freedom $N_{\text {dof }}$, respectively. As expected, he asymptotic convergence of $e_{\mathrm{en}}$ with respect $h$ coincides with the one predicted by the theory. Figure 5 show the convergence of the difference between the total electrostatic energy $E$ and the estimated one $E_{h}$ with respect to the computational time required. From this figure we can conclude that the method is convenient even if one is willing to invest in the solution only a few seconds. We should also mention that the advantage is even bigger in practice given that with high order one uses 


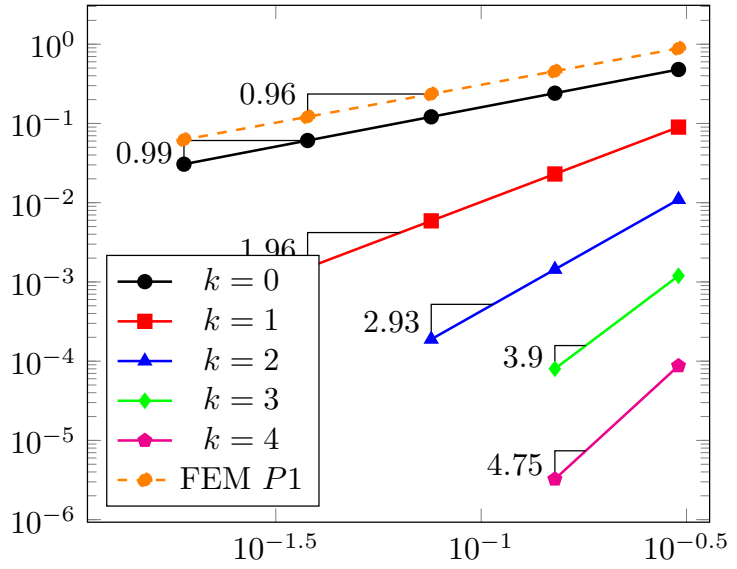

Fig. 3: $e_{\mathrm{en}}$ vs. $h$.

less elements and thus saves a lot of time also by reducing the meshing time (which is not included in this analysis).

For the benchmark we used tetrahedral meshes: on one hand to be able to compare with the classical finite element method and, on the other hand, because many efficient, automatic and open source mesh generators for such elements exist and they are well-integrated in the computer-assisted modeling chain. Moreover, the convergence slopes are correctly retrieved only if the set of meshes are produced by recursively subdividing the coarsest one. Now this is quite difficult to perform with general polyhedral meshes because the appropriate tools are currently being developed.

To validate the method also with general polyhedral elements, we start from an initial mesh composed of tetrahedral elements which is fine enough to capture the geometric features of the domain (for example, curved boundaries) and the scales of the exact solution. Then, we perform the computations on a polyhedral mesh obtained by agglomerating elements of the background tetrahedral mesh. Doing so we obtain for example the mesh with 470 polyhedra represented in Fig. 6. The correct solution obtained with $\mathrm{MHO}$ and $k=1$ is represented in Fig. 6. A similar technique has been used in the adaptive coarsening strategy [3], which is a new procedure that is able to drastically reduce the number of degrees of freedom with respect to the ones resulting from the tetrahedral background mesh without the need to regenerate a new mesh.

\section{REFERENCES}

[1] L. Beirão da Veiga, A. Ern, Preface to the special issue Polyhedral discretization for PDE, ESAIM: M2AN, Vol. 50, No. 3, pp. 633-634, 2016.

[2] L. Codecasa, R. Specogna, F. Trevisan, A New Set of Basis Functions for the Discrete Geometric Approach, J. Comput. Phys., Vol. 229, pp. 74017410, 2010

[3] D.A. Di Pietro, R. Specogna, An a posteriori-driven adaptive Mixed HighOrder method with application to electrostatics, J. Comput. Phys., Vol. 326, pp. 35-55, 2016.

[4] D.A. Di Pietro, A. Ern, Arbitrary-order mixed methods for heterogeneous anisotropic diffusion on general meshes, IMA J. Numer. Anal., 2016, in press.

[5] R. Specogna, One stroke complementarity for Poisson-like problems, IEEE Trans. Magn., Vol. 51, No. 3, 7401404, 2015.

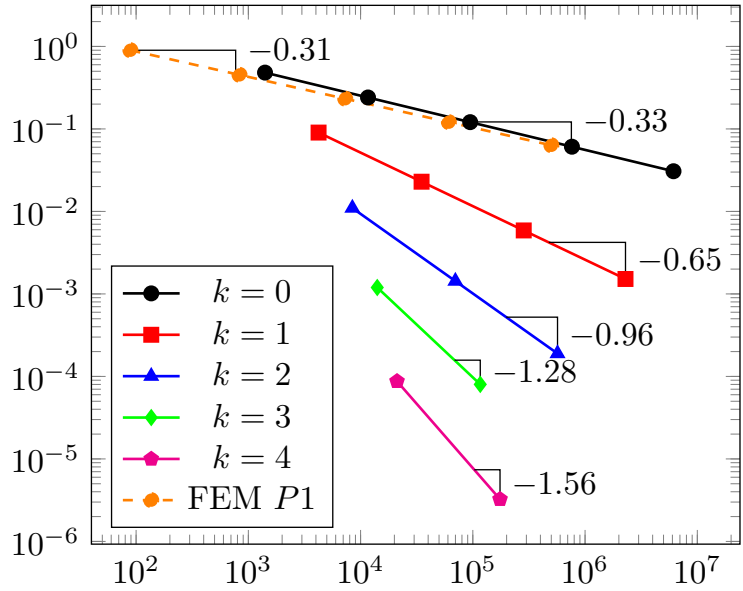

Fig. 4: $e_{\mathrm{en}}$ vs. $N_{d o f}$.

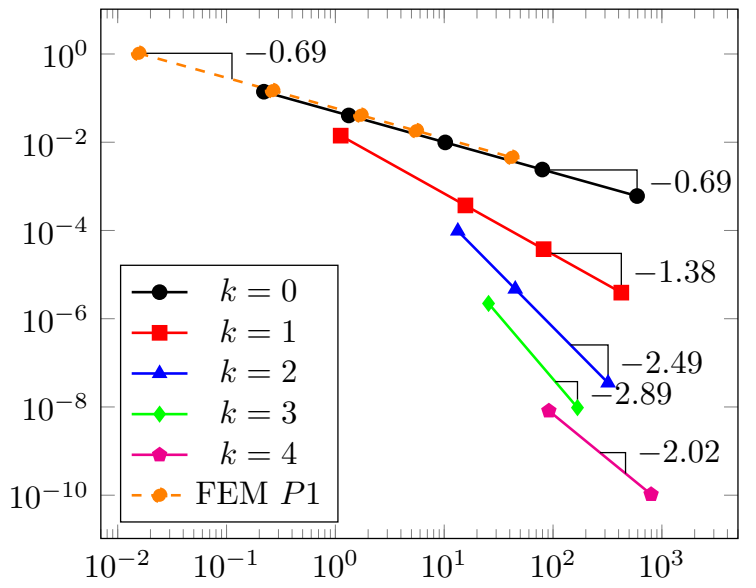

Fig. 5: $E-E_{h}$ vs. total wall time in seconds.
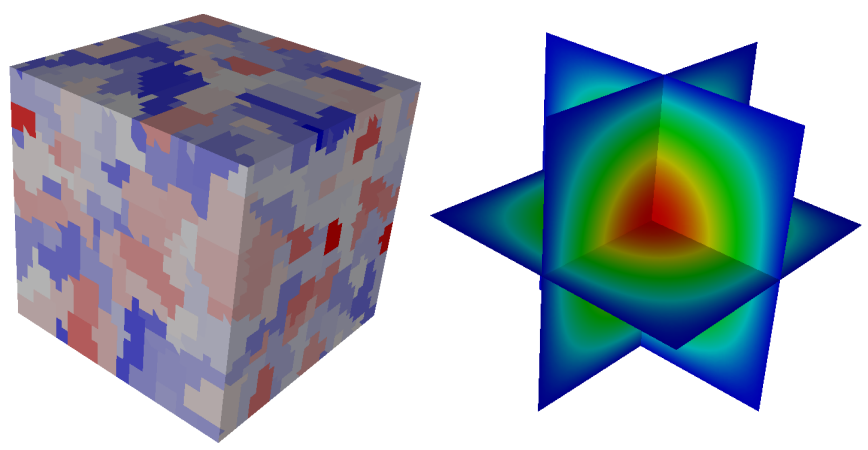

Fig. 6: On the left, the polyhedral mesh used in the computation composed by 470 polyhedral elements. Each different color represent a polyhedral mesh element obtained by gluing a set of tetrahedra belonging to a background mesh consisting of about 270000 tetrahedra. On the right, the result in terms of potential on three slices of the domain for $k=1$.

[6] D.A. Di Pietro, A. Ern, Mathematical Aspects of Discontinuous Galerkin Methods, No. 69 in Mathematics \& Applications, Springer, Berlin, 2012.

[7] J. Aghili, S. Boyaval, D. A. Di Pietro, Hybridization of mixed highorder methods on general meshes and application to the Stokes equations, Comput. Meth. Appl. Math., Vol. 15, No. 2, pp. 111-134, 2015.

[8] Y. Notay, An aggregation-based algebraic multigrid method, Electron, Trans. Numer. Anal, Vol. 37, No. 6, pp. 123146, 2010. 